\title{
Patočka et la dialectique de l'apparaître
}

\section{Chiara Pesaresi}

\section{(2) OpenEdition}

\section{Journals}

Édition électronique

URL : https://journals.openedition.org/alter/1877

DOI : $10.4000 /$ alter. 1877

ISSN : 2558-7927

\section{Éditeur :}

Association ALTER, Archives Husserl (CNRS-UMR 8547)

\section{Édition imprimée}

Date de publication : 1 novembre 2019

Pagination : 65--81

ISBN : 978-2-9550449-5-7

ISSN : 1249-8947

\section{Référence électronique}

Chiara Pesaresi, « Patočka et la dialectique de l'apparaître », Alter [En ligne], 27 | 2019, mis en ligne le 22 décembre 2020, consulté le 13 juin 2021. URL : http://journals.openedition.org/alter/1877 ; DOI : https://doi.org/10.4000/alter.1877

Ce document a été généré automatiquement le 13 juin 2021.

Revue Alter 


\title{
Patočka et la dialectique de l'apparaître
}

\author{
Chiara Pesaresi
}

Redécouvrir la dialectique malgré soi est peut-

être une plus sûre manière de la prendre au sérieux que de commencer par elle, d'en savoir

d'avance la formule ou le schéma, et de l'appliquer partout [...] sans se demander d'où vient que l'être soit dialectique ${ }^{1}$.

1 Parmi les questions cruciales soulevées par Patočka, il y a certainement celle du rapport entre monde et existence : d'après l'auteur, ce rapport peut être décrit dans les termes d'un mouvement «au double volet» dont la critique a remarquablement

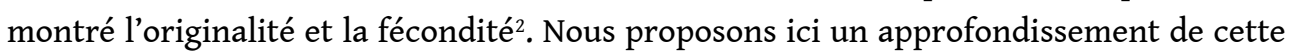
question par la mise en lumière de l'aspect dialectique de tout mouvement premièrement, de celui de l'apparaître en tant que mouvement d'individuation et de détermination de l'étant; mais également du mouvement de l'existence dans sa triple articulation interne, ainsi que dans son rapport au proto-mouvement.

2 Toute au long de son œuvre, Patočka aborde - de façon plus ou moins explicite et sur des plans différents (phénoménologie du mouvement, philosophie de l'histoire, théorie des trois mouvements de l'existence) - la question de la dialectique : il suffit de penser à la définition de l'esprit comme « dialectique de l'affection négative ${ }^{3}$ donnée en 1949 , ou à l'hypothèse de 1969 d'une "dialectisation de la phénoménologie " ${ }^{4}$. Les références les plus significatives se trouvent toutefois dans son ouvrage de 1964, Aristote, ses devanciers, ses successeurs, et notamment dans le dernier chapitre, consacré à Descartes et Hegel : Patočka parle ici du mouvement comme unité processuelle, "altérité qui se surmonte, indifférence qui se supprime ${ }^{5} »$. Ces expressions demandent sans doute une élucidation du rapport entre mouvement et dialectique et invitent en même temps à une explicitation du dialogue, souvent méconnu, que Patočka entretient avec la pensée hégélienne ${ }^{6}$. Celle-ci, venant se greffer sur la phénoménologie d'un côté et sur l'héritage grecque de l'autre, nous apparaît en effet comme l'une des sources de la 
définition patočkienne du mouvement en tant que medius terminus entre monde et existence.

3 Nous essayerons de montrer la pertinence et l'intérêt de ces questions en trois moments. Prenant le départ de la définition de l'esprit comme "dialectique de l'affection négative ", il s'agira de déceler la structure dialectique de l'apparaître en tant que dynamisme universel de la physis. Ensuite, nous tenterons de démontrer que le caractère négatif et inactuel, qui fait de l'esprit et de la proto-manifestation des mouvements dialectiques, définit également le mouvement de l'existence humaine, et cela en un double sens: d'un côté, il la détermine "extérieurement", en tant qu'existence individuée se séparant de la totalité générative du monde ; de l'autre côté, il la définit "intérieurement", en tant que mouvement tripartite d'enracinementprolongement-percée. Il s'impose enfin un questionnement plus large sur le rapport entre phénoménologie et dialectique et sur sa pertinence au sein de la réflexion patočkienne? ${ }^{7}$.

\section{De la « dialectique de l'affection négative » à la dialectique de la physis}

4 Pour comprendre pourquoi le mot "dialectique » désigne ce double caractère du mouvement, négatif (auto-négateur) et possibilisant (inactuel) à la fois, il faut remonter au texte de 1949, Éternité et historicité. Ici, Patočka définit l'esprit comme une "dialectique de l'affection négative " $^{8}$. Dès son émergence au sein du réel, l'êtrehumain ne peut en effet se manifester que comme une différence et, plus radicalement, comme une protestation, un conflit avec tout ce qui est actuellement donné, en vue de son dépassement. La structure dialectique de l'esprit tient alors en son caractère essentiellement négatif face au donné et toujours projetée dans un horizon de possibilité (c'est-à-dire dans le domaine du non-actuel). Cette expression, «dialectique de l'affection négative ", désignerait alors un mouvement de transcendance négative, qui s'opère en même temps vis-à-vis, contre et dans la totalité des choses 9 .

5 Cette dynamique de négation et de projection, de dépassement vers le non-actuel, estelle une prérogative strictement humaine ? Ou bien ces deux aspects du mouvement de l'esprit, à savoir sa structure éminemment dialectique, appartient également au mouvement général du monde, à cette totalité asubjective et omni-englobante dite physis dont l'esprit lui-même surgit et se sépare ? À ces questions, Éternité et historicité donne déjà une esquisse de réponse: en effet, Patočka y dit clairement qu'une dialectique singulière serait « inhérente à la structure essentielle de l'esprit et, par là, à la nature même de la réalité $»^{10}$. Or, cette réalité sera ensuite désignée comme totalité dynamique du monde ou physis. Au cours des années soixante, Patočka opère en effet une radicalisation de l'idée de monde naturel en termes de physis au moyen d'une analyse du mouvement. Dans sa Méditation de 1969, le monde est le milieu universel et omni-englobant de l'individuation de l'étant :

Ne sera-t-il pas possible ainsi d'introduire dans le contexte ontologique le monde au sens fort du terme, le monde existant de manière autonome? L'ancienne $\varphi v ́ \sigma \iota \zeta$ ne sera-t-elle pas restituée en tant qu'arché, gouvernant tout le singulier ${ }^{11}$ ?

6 Avant d'être un corrélat ou un horizon, le monde est donc l'événement permanent qui amène l'étant à la présence et à sa déterminité. Cette manifestation sans destinataire, coïncidant avec l'émergence des étants individués, renvoie à la conception grecque du 
monde en tant que physis, au «monde des anciens, [...] inépuisable, négatif, mystère, enveloppé $~^{12}$. La sagesse présocratique découvre en effet le monde comme un "drame primordial, proto-mouvement et processus originaire " qui provoque "l'émergence hors de la nuit du non-être, l'union qui crée entre la naissance et la mort un espace à couvert où des êtres liés par une dépendance réciproque font place les uns aux autres et derechef se suppriment $»^{13}$. L'ouverture de l'horizon du monde et l'installation en lui d'une existence reposent alors sur une ouverture préliminaire : celle de la physis en tant que principe dynamique, archè - au sens de ce qui détermine dans la continuité et régit en permanence - de l'être et de l'apparaître ${ }^{14}$. Cela revient à dire qu'il faut remonter en deçà de la relation entre mondain et intramondain (ce qui existe sur le fondement du monde), vers l'événement à partir duquel un monde existe et s'ouvre. Le monde «au sens fort du terme » est donc repensé sous le registre du mouvement et de l'événement, la physis étant précisément l'événement de l'essence ${ }^{15}$.

7 Il s'agit maintenant de montrer pourquoi ce drame primordial n'est pensable qu'en termes dialectiques et quels sont les passages conceptuels qui amènent à une telle interprétation. Dans son grand ouvrage de 1964, Aristote, ses devanciers, ses successeur, Patočka retrace les différentes conceptions du mouvement dans l'histoire de la philosophie, des Présocratiques jusqu'à Hegel. C'est notamment sur les acquis de la Physique d'Aristote (livre II) qu'il s'appuie pour dévoiler le mouvement comme « facteur ontologique fondamental $\aleph^{16}$. Le soustrayant à sa définition simplement quantitative, Aristote a en effet le mérite d'avoir montré le mouvement comme principe de l'être des étants ${ }^{17}$.

8 Malgré cela, le mouvement aristotélicien demeure, aux yeux de Patočka, susceptible de deux critiques majeures. En premier lieu, il suppose toujours un substrat immuable, et donc immobile, comme sa condition préalable. Le mouvement se réduirait ainsi à la simple alternance des contraires sur un substrat; autrement dit, il ne serait qu'un changement d'état. Dans le mouvement aristotélicien, affirme Patočka, il y a " quelque chose qui se maintient, qui demeure inchangé, le changement étant défini par rapport à ce constant $\aleph^{18}$. En second lieu et conséquemment, le mouvement ne concerne que le monde sublunaire, à savoir les étants naturels, la teneur d'essence restant immobile. Or, ces deux aspects problématiques s'expliquent par la subordination du mouvement

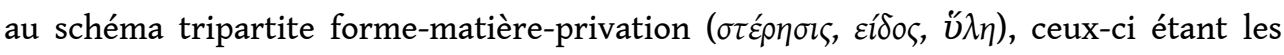
trois archai qui gouvernent tout mouvement ${ }^{19}$.

9 À la réduction du mouvement à l'immobile et à sa limitation au seul étant, Patočka oppose un mouvement "performatif», ontogénétique, qui constitue sa propre unité (son propre "être») et celle de la chose en mouvement; en d'autres mots, un mouvement comme principe de l'être, de l'individuation et de la détermination de l'étant. La dynamisation du substrat correspondrait alors, plus radicalement, à la désubstantialisation du mouvement : celui-ci devient la condition de possibilité de la constitution (et non seulement de la détermination) du « substrat » lui-même, tout en y demeurant irréductible. En ce sens, c'est la dialectique de génération et de corruption

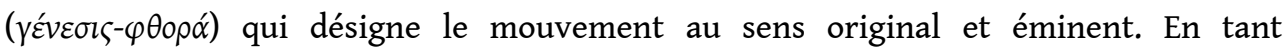
qu'événement de l'essence, mouvement dialectique « d'émergence et de disparition de l'étant ", la physis demeure irréductible à la simple présence des choses, ainsi qu'à un substrat ou à la simple alternance des contraires sur un substrat. Comment une telle radicalisation s'opère-t-elle? 
10 Dans des notes de travail de 1969 intitulées Phénoménologie et ontologie du mouvement, Patočka souligne la difficulté d'éviter toute réduction du mouvement à l'immobile, problème déjà abordé en 1964. En raison de l'absence de tout substrat comme référence première et ultime du mouvement, sa réduction à l'immobile est en effet, écrit Patočka, tout à la fois nécessaire et impossible, inachevable ${ }^{20}$. C'est à partir de ce constat qu'il est amené à qualifier de négative la définition du mouvement : en raison de cette nécessitéimpossibilité de la réduction, la pensée du mouvement n'est pas une pensée positive mais " une pensée dialectique, négatrice $~^{21}$. Le mouvement n'est plus définissable, dès lors, au moyen des trois principes logiques établis par Aristote car, à la place de ce mouvement "à même quelque chose ", l'on suppose plutôt que ce quelque chose est sa possibilité, qu'il n'y a en lui rien avant les possibilités et sous-jacent à celles-ci ${ }^{22}$. Si l'on refuse donc toute re(con)duction du mouvement à une permanence, on ne peut que le penser et le définir par sa possibilité, à savoir par sa non-actualité. Par conséquent, ce n'est que sur un plan plus profond que le plan catégorial (plan de l'étant présent, actuel) qu'on peut saisir ce qu'est le mouvement dans son fond. Or ce plan aurait justement la particularité de "prendre en vue l'affirmation et la négation ${ }^{23}$ ", à savoir

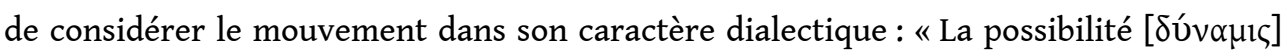
simultanée du contraire de ce qui détermine actuellement la ǔ $\lambda \eta$, fait ainsi que la ǔ $\lambda \eta$ n'est jamais pleinement ce qu'elle peut être, à savoir qu'il y a un "non" autre que la négativité de la $\sigma \tau \dot{p} p \eta \sigma \iota \zeta . ~{ }^{24}$

11 La négativité originaire du mouvement ne réside donc ni dans la privation, ni dans le substrat matériel, ni dans l'alternance des contraires (changement), mais plutôt dans ce

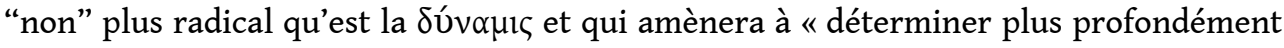
l'élément négatif initialement contenu dans la ǔ $\lambda \eta »^{25}$. De ce fait, la définition du mouvement au moyen de la $\delta u ́ v \alpha \mu \iota$ est une définition négative, « une définition par le non-être qu'est le "pas encore". L'être actif, l'acte de l'être-encore-non-être = le mouvement ${ }^{26}$. Autrement dit, l'auto-négation impliquée dans le mouvement est inséparable de son caractère de possible. En outre, le "non-encore" montre non seulement l'aspect négatif et inactuel du mouvement, mais aussi son caractère temporel ou protensif, qui manque au schéma tripartite en raison de sa relativité à un substrat immobile et par là intemporel.

Soustrait aux principes logiques et donc à sa référence à un substrat préalable - c'est-àdire désubstantialisé - le mouvement peut être ramené à son sens originaire d'« entrée dans la singularité ». Toute détermination est ainsi « un devenir dans l'indéterminé et en vertu de sa puissance; aussi cet indéterminé est essentiellement dynamique, événement et temps avant tout ${ }^{27}$.

Nous avons essayé de démontrer que c'est en vertu de son caractère dialectique que le mouvement est, chez Patočka, performatif et événementiel. À l'instar de la pensée grecque et, comme on le verra, hégélienne, la continuité du processus ne s'identifie pas à un continuum monotone, mais plutôt à une Aufhebung perpétuelle, qui trouve son expression dans l'événement de l'individuation. Si l'esprit est une dialectique (de l'affection négative) et si cette dialectique est synonyme d'une instance négative et inactuelle; et si, de surcroît, on retrouve cette double instance dans le mouvement de manifestation, ne pouvons-nous alors parler à raison d'une dialectique de l'apparaitre? 


\section{La dialectique hégélienne comme relation interne} principe non seulement ontologique mais ontogénétique, par lequel «le monde vient à l'être ${ }^{28}$ " : l'idée de physis traduit alors ce dynamisme de l'indéterminé, dominé par un principe dialectique de génération et de corruption qui reste indéfiniment ouvert. Autrement dit, Patočka (re)découvre une dialectique interne au devenir cosmologicophysique, qui ne se définit et qui ne peut être saisie que négativement et au moyen du non-actuel ou de sa possibilité. Une telle idée de dynamisme universel, nous venons de le voir, est décelable dans la première philosophie grecque, qui a le mérite d'avoir pensé le mouvement comme entrée dans la présence des choses individuées. Il nous semble toutefois que le dernier chapitre de l'Aristote, consacré à Descartes et Hegel, démontre qu'une telle redéfinition du monde et du mouvement relève également de la reprise critique de la dialectique hégélienne. D’après Patočka, celle-ci « trouve en effet dans le concept aristotélicien de mouvement un prologue grandiose ${ }^{29}$.

Présocratiques et, dans la modernité, avec Hegel, qu'on peut penser le mouvement non comme «mouvement dans le monde, mais comme mouvement du monde lui-même " ${ }^{30}$. L'héritage hégélien nous semble donc jouer un rôle clé, dans la mesure où il permet précisément de :

[...] résoudre, sur une base nouvelle, le problème qui fut celui d'Aristote: le problème du mouvement conçu de manière ontologique, du mouvement qui n'est pas une simple relation toute faite, résultat d'une constitution, mais cela même qui constitue l'être des étants qui sont en mouvement et en devenir, non seulement extérieurement et relativement, mais par tout ce qu'ils sont ${ }^{31}$.

L'être est animé par un mouvement interne qui en constitue l'unité et qui, en même temps, installe l'étant dans la présence, le fait entrer dans l'apparaître. Or, c'est précisément la dialectique hégélienne qui reçoit, dans le dernier chapitre de l'Aristote, la définition de relation ou de mouvement interne :

Le concept hégélien de relation, en tant qu'unité du scindé, parenté de l'étranger, concordance du divers, permet-il de couvrir l'immense amplitude de variation des mouvements les plus hétérogènes et les plus diversement gradués que l'on rencontre dans la nature extra-humaine, chez l'homme et dans la société humaine : autorisant au même titre la plus extrême altérité (Fremdheit) et la parenté la plus étroite des membres de la relation, le mouvement est alors précisément l'unité en tant que processus, l'altérité qui se surmonte, l'indifférence qui se supprime ${ }^{32}$.

Par le fait qu'il montre la relation - et notamment la relation d'Aufhebung, de suppression et de conservation - comme quelque chose de vivant, le mouvement dialectique peut représenter le principe d'unité des dimensions de l'être, à savoir du devenir du monde et de l'existence humaine comme faisant partie de ce devenir. Grâce à la dialectique, dont la tâche est de "montrer la relation comme quelque chose d'interne et de vivant ${ }^{33}$, l'étant n'est jamais isolé mais toujours pris dans un contexte relationnel ; en ce sens, l'unification est l'essence du mouvement dialectique hégélien.

En d'autres mots, Hegel fait de la dialectique le principe de l'unité dynamique de l'être et de l'apparaître, et du négatif l'âme motrice de ce progrès ; à travers la dialectisation stricto sensu de la totalité, il opère alors une véritable radicalisation du mouvement aristotélicien, en montrant, « de façon bien plus conséquente et plus profonde que ce n'était le cas chez Aristote, que l'être de l'étant est mouvement $\aleph^{34}$. Pour Hegel, la 
totalité n'est en effet pensable que du point de vue du mouvement, qui décrit avant tout le devenir du cosmos et seulement dans un sens dérivé le devenir dans le cosmos ${ }^{35}$. $\mathrm{Au}$ moyen d'une transposition phénoménologique de la dialectique hégélienne - soustraite, certes, à ses issues métaphysiques - Patočka est donc au gré de dépasser les limites, évoquées ci-dessus, de la conception aristotélicienne du mouvement.

Une telle interprétation de la dialectique témoigne d'une certaine continuité avec la lecture kojèvienne. En 1947, Patočka reçoit le texte de Kojève et s'y réfère constamment dans son cours de 1949-1950 sur la Phénoménologie de l'esprit. Dans sa célèbre Introduction, Kojève insiste sur le fait que Hegel est le premier philosophe à avoir soustrait la dialectique au domaine de la seule question méthodologique pour en faire "la nature propre, vraie, des choses mêmes " ${ }^{36}$. Pareillement, la dialectique ne définit pas pour Patočka un procédé logique, mais la structure même de l'être, de sa réalisation et de sa manifestation. La phénoménologie hégélienne représenterait alors «l'exposition de la dynamique universelle de l'esprit» : elle est la description moins d'une réalité donnée que de son mouvement de formation et de manifestation. On pourrait dire que l'identité $d u$ monde et du mouvement de l'apparaître traduit phénoménologiquement, chez Patočka, l'identité hégélienne de l'esprit et de sa manifestation mondaine.

Il nous semble alors que l'originalité de l'idée patočkienne d'un dynamisme universel qui est mouvement, événement et phénomène à la fois, et qui rend raison de l'émergence d'une vie individuée et d'une conscience en son sein - relève de la reprise critique, à travers le prisme de la phénoménologie, de la Physique d'Aristote, de la Phénoménologie de l'esprit de Hegel et de la sagesse présocratique. Bien sûr, Patočka souligne l'impossibilité de souscrire à la thèse de Hegel sur l'esprit comme absolu ainsi que l'illégitimité de la reconduction du dynamisme universel au dynamisme subjectif. Cela n'empêche pas de reconnaître chez Hegel un mouvement ontologique et performatif qui, en tant que relation interne, rend compte non seulement de l'apparaître du monde mais aussi du surgissement d'une conscience. Hegel a le mérite d'avoir montré que ni l'existence ni la conscience ne sont données a priori ou immédiatement, raison pour laquelle elles demandent un mouvement pour se conquérir - mouvement de négation de la simple extériorité ou, pour le dire avec Patočka, de l'objectité actuellement donnée.

21 À ce moment de notre questionnement, nous pouvons constater que tout mouvement humain (esprit) et cosmique (physis) - a révélé ces deux caractères essentiels : d'un côté, une non-coïncidence de l'être avec soi et, de l'autre côté, une tension au-delà de toute actualité. Ces deux déterminations, négativité et inactualité, convergent dans une définition dialectique du mouvement.

\section{La dialectique sans synthèse des trois mouvements et l'événement de la liberté}

Cette dynamique universelle d'individuation et de détermination de l'étant pose la question du rapport entre monde et existence, c'est-à-dire entre le mouvement dialectique de l'apparaître et le mouvement qui nous est propre. Il s'agit à vrai dire d'une double question, car elle renvoie, d'un côté, au surgissement d'une vie humaine individuée au sein de la manifestation primaire et, de l'autre côté, à sa différence radicale, à sa «transcendance négative » qui en brise la continuité. Le mouvement 
peut-il articuler les deux sens de l'apparaître? Autrement dit, peut-il représenter la commune mesure entre l'homme et le monde ${ }^{37}$ ? Nous nous attacherons à montrer que, si le mouvement est au gré d'une telle médiation, ce n'est qu'en vertu de son caractère dialectique, que nous avons découvert au cœur de la proto-manifestation. L'hypothèse est alors la suivante: non seulement cette manifestation primaire, mais aussi le mouvement propre à l'homme (en tant que liberté finie), relève d'une dialectique qui montre l'impossibilité de toute synthèse.

Tout d'abord, l'existence humaine s'inscrit, par son individuation, dans le dynamisme universel de la physis : nous sommes donc pris, englobés dans la dialectique originaire du fond indifférencié et de l'étant déterminé. Nous faisons partie de ce «drame glorieux [...] non pas en tant que spectateurs, mais en ce sens, que nous-mêmes, comme les choses, sommes dépensés pour et consommés dans ce procès ${ }^{38}$ ». Mais si notre individuation s'intègre indifféremment à cet univers de déploiement de l'étant, le mouvement existentiel qui nous est propre en diffère radicalement : la séparation de la totalité est chez l'homme le drame d'une « expulsion originaire de la partie hors du tout $\aleph^{39}$, ce qui fait de l'homme un étant essentiellement incomplet. Il est en effet différent au sens étymologique du terme " différer ", du latin dis-ferre, tenir séparé. Déjà Hegel définit l'homme comme un animal malade : alors que « la vie animale est une vie dans l'unité, intégrée sans discontinuité dans tout le reste de la nature $»^{40}$, la vie humaine s'écarte du continuum dynamique et génératif de la physis comme une différence qui le transperce. Le surgissement de l'individu humain est donc un événement dans l'événement, qui vient «troubler la paix de la nature indifférente, la paix de l'indifférence envers l'être propre, et s'écarte de l'ordre antérieur du monde ${ }^{41}$. La genèse de la vie marque déjà une discontinuité dans le dyna-misme de la physis; cette discontinuité entrainée par toute individuation se dédouble pour l'étant humain, puisque son surgissement est de l'ordre d'une installation conflictuelle. La dialectique d'appartenance et de différence qui articule le rapport entre vie individuée et monde, aboutit chez l'homme à l'événement de la libertée ${ }^{42}$. Dans les écrits phénoménologiques des années soixante, la protestation de l'existence face au monde en totalité protestation qui, comme on l'a vu, était le propre de l'esprit - s'achève et s'intègre dans le troisième mouvement, le mouvement de percée. Sous la figure de ce troisième mouvement, qui marque l'entrée dans l'histoire, Patočka nous semble ainsi réintégrer l'idée de liberté telle qu'elle avait été formulée dans les années quarante et cinquante, notamment dans Éternité et historicité et Platonisme négatif. Ici, Patočka avait montré que la réduction phénoménologique relève d'un acte de liberté et que, corrélativement, cette liberté se manifeste comme négation du donné et transcendance :

Nous considérons à la fin la démarche réductive comme faisant partie d'une dialectique singulière, inhérente à la structure essentielle de l'esprit et, par là, à la nature même de la réalité, dialectique qui se manifeste en ceci que chaque tentative pour sortir de son cadre nous y ramène, la ressuscite. L'essence de cette dialectique est la lutte de l'esprit avec l'objectité. Quand cette étrange objectité - le sujet, l'esprit, la conscience - surgit au sein de la réalité, elle ne peut que protester contre celle- $\mathrm{ci}^{43}$.

Ces acquis seront intégrés, à la suite des recherches sur le mouvement et sur l'apparaître, dans la théorie des trois mouvements de l'existence. Patočka montre ici que c'est en raison de sa séparation « traumatique » de la totalité que l'existence surgit comme un conflit, contre le monde et pour le monde à la fois. Contre le monde, en tant que lutte et insatisfaction face à la totalité actuelle du donné ; et pour le monde, en tant 
que tendance à se réapproprier soit d'une origine primordiale à laquelle elle a été arrachée, soit d'une totalité de sens qui lui échappe à cause de sa finitude. Dans le troisième mouvement de l'existence, la négation de l'individualité demandée par l'individuation elle-même prend la forme d'une liberté comprise comme "praxis historique et dialectique ", c'est-à-dire installée de façon problématique dans un monde commun ${ }^{44}$. Ce n'est que la prise de conscience de sa condition de déracinement qui peut ouvrir l'existence à ses possibilités fondamentales. Mais l'acquis principal de la théorie des trois mouvements par rapport aux années quarante nous semble tenir en ce que la liberté, dans son caractère négatif et protensif à la fois, est enfin montrée comme le pli qui dédouble la dialectique de la manifestation primaire. L'inclusion de la vie humaine dans le processus d'individuation entraîne le mouvement aussi bien originaire mais contraire de libre insertion de l'existence dans le monde - insertion qui ne se fait que sur la base d'une expulsion originaire. Cela a deux conséquences majeures :

1. «Tout rapport libre est nécessairement un conflit ${ }^{45}$ : autrement dit, ni l'émancipation du besoin ni l'ouverture libre au monde naturel, communautaire et historique, amènent à une synthèse au gré de résorber le conflit dans la stabilité du positif; de même, le dépassement de la naturalité de l'existence n'est jamais achevé ni acquis. La liberté représente en effet une "solution conflictuelle » et constamment menacée par la possibilité de la déchéance. Selon un "dépassement dialectique (toutefois toujours temporaire) de la polarité du conflit $»^{46}$, l'existence tend, dans son mouvement global, à la réalisation d'une majeure "positivité existentiale", qui pourtant ne dissipe pas l'élément conflictuel qui en est le moteur.

2. La structure dialectique et ouverte du mouvement de l'existence empêche de penser le troisième mouvement comme abandon définitif de la situation vitale, d'enracinement, dont le premier mouvement est l'expression. Les trois mouvements de l'existence sont dans un rapport de suppression et de conservation réciproque qui montre que la dimension naturelle de l'existence représente en même temps le fondement de la liberté et le fond qui la menace. Les trois mouvements sont cooriginaires et simultanés, ce qui veut dire que chacun présuppose les deux autres. Cette co-implication est possible dans la mesure où, dans chaque mouvement, est dialectiquement contenu le principe de son propre dépassement et de sa conservation. Car, comme le souligne Patočka à plusieurs reprises, l'être en mouvement se caractérise par le fait "de ne pas être ce qu'il est», d'exclure toute identité ou coïncidence avec soi, à savoir d'être internement traversé par la négativité et par la possibilité. La dialectique hégélienne nous apprend précisément que toute détermination, dans son identité, a un caractère auto-négateur qui représente le moteur interne de son propre dépassement. Dans cette perspective, le poids du besoin, la contraposition sociale et la lutte pour l'éveil représentent autant de figure de l'Aufhebung.

La dialectique des trois mouvements fait ainsi écho à la dialectique entre physis et existence, sous la figure d'une constante oscillation entre une situation primordiale d'insertion "passive» et une impulsion contraire au redressement. L'élément dialectique, à savoir négatif et possibilisant, définit alors non seulement a) la structure et la dynamique interne à chaque mouvement, mais aussi b) la relation des trois mouvements entre eux et c) celle du mouvement général de l'existence à l'égard du tout du monde, comme séparation et «dialectique de l'affection négative ». En bref, c'est la trame du mouvement tout court qui s'avère foncièrement dialectique. Il est significatif, en ce sens, ce que Patočka écrit sur Hegel dans sa Méditation: 
Il se peut que la philosophie hégélienne de l'esprit subjectif contienne, dans la figure de la conservation et du dépassement, toute la "philosophie de la vie" de son époque. La "marche du devenir" dont part la philosophie de l'esprit subjectif signifie que la base naturelle qui se meut en direction de son but est néanmoins toujours conservée dans son dépassement ${ }^{47}$. y a un rapport d'Aufhebung, de suppression et de conservation à la fois, grâce auquel le mouvement de la vie ne se renferme pas sur soi-même mais reste ouvert ${ }^{48}$. Patočka utilise à ce propos la métaphore du double cercle de la vie et de l'existence ${ }^{49}$ : cette métaphore signifie que le mouvement de l'existence humaine ne relève pas de, ni ne s'achève dans une essence figée. On pourrait penser plutôt à une spirale, qui reste indéfiniment ouverte tout en revenant, différemment mais constamment, sur soimême; car notre racine vitale n'est jamais véritablement dépassée, ni notre liberté acquise une fois pour toutes.

Dans la théorie des trois mouvements de l'existence, la liberté en tant que surgissement et protestation de "l'humain » est donc montrée comme le pli d'une appartenance radicale à la physis, ce qui empêche de penser l'acte de liberté comme un acte absolu : le monde n'est pas «un produit de la liberté mais simplement ce qui rend possible une liberté finie $»^{50}$. L'installation historique de l'homme dans le devenir cosmologique est un double événement dialectique, ce qui permet d'esquiver tout idéalisme ou hypostase de la subjectivité. En même temps que conditionnée par l'apparaître du monde, la liberté est en effet condition de toute manifestation : autrement dit, l'animator ${ }^{51}$ de la liberté est le monde, mais celui-ci n'arrive à sa clarté que par le geste de liberté qui le manifeste. Le dynamisme dialectique de l'apparaître articule donc une philosophie de la totalité et une philosophie de la liberté et ces deux principes sont réconciliés au moyen du mouvement dialectique, à savoir à la condition de les expliquer comme des «principes négatifs».

\section{Conclusions}

\section{Dialectique et phénoménologie}

Nous avons essayé de montrer que l'articulation des deux événements qui sont l'individuation de l'étant et l'existence humaine n'est possible que sur la base d'un principe dynamique et dialectique. En tant qu'essentiellement auto-négateur et inactuel, le mouvement est à la fois la condition d'une appartenance indépassable et d'une différence radicale entre monde et existence. Une telle articulation des plans anthropologique et cosmologique nous a paru relever d'une reprise en filigrane de la dialectique hégélienne en tant que mouvement ontologique et relation interne. Patočka interprète en effet la dialectique de manifestation de l'esprit comme une analyse de l'apparaître au sens phénoménologique. À travers une lecture critique, influencée par l'interprétation kojèvienne, de la dialectique réelle de la Phénoménologie de l'esprit, Patočka ouvre ainsi la voie à une dialectique phénoménologique, dépourvue de toute synthèse positive, se décelant au niveau de l'apparaitre et de l'existence. Cette dialectique ne serait pas mue par une négation formelle ou logique, mais exprimerait au contraire la «contradiction où l'infini lui-même passe à travers le désert » du fini ${ }^{52}$. Non pas une dialectique du concept, donc, mais « un mouvement de l'être » auquel l'homme « doit répondre par sa fidélité à l'énigmatique » ${ }^{53}$. 
32 La dialectique pourrait ainsi exprimer la pensée de l'absolu, mais en tant que « correspondant à l'appel de l'être »; et cet appel devrait «s'exercer en premier lieu contre la subjectivité close $»^{54}$. En ce sens, la dialectique pourrait devenir la voie d'une phénoménologie asubjective et alors « phénoménologie et dialectique [pourraient] aller de pair $»^{55}$. Cette "dialectisation de la phénoménologie $»^{56}$ implique (et même trouve son fondement dans) une définition négative et in-actuelle du mouvement du monde et de l'existence. Là où la phénoménologie demeure insuffisante - à savoir dans la considération de l'aspect dynamique et historique de la réalité - c'est une dialectique soustraite aux schémas métaphysiques qui rend raison du dévoilement des étants et des structures générales de l'être ${ }^{57}$. Par différentes étapes, nous avons essayé de faire émerger cet aspect dialectique de tout mouvement : du monde en tant que mouvement ontogénétique, de l'existence en tant que mouvement tripartite et de leur "corrélation ». C'est en effet la dialectique qui rend compte à la fois: a. d'un commencement qui n'est pas une création ex nihilo ${ }^{58}$, mais qui ne demeure pas moins une institution d'existence et de sens; b. d'un mouvement indéfiniment ouvert et intotalisable.

L'irréductibilité du mouvement et le caractère inachevé de l'existence impliquent l'impossibilité foncière de toute synthèse positive et l'installation permanente "dans l'irréconcilié, dans l'ouverture à ce qui ébranle, autrement dit dans le conflit et la lutte, [...] vers un possible dont la réalisation n'est pas donnée par avance ${ }^{59}$. C'est le négatif qui vient briser toute initiative de totalisation à la faveur d'une totalité phénoménale, dynamique et historique. L'Aufhebung de la métaphysique traditionnelle se joue dans cette distinction entre totalisation et totalité : la phénoménologie doit, d'un côté, se soustraire au systématisme totalisant des représentations métaphysiques et, d'un autre côté, revenir à la « volonté philosophique essentielle » de la métaphysique en tant que problématisation de l'horizon actuel et questionnement sur la totalité ${ }^{60}$. Cette totalité, chez Patočka, n'est ni une abstraction conceptuelle ni une indifférenciation spatiotemporelle, mais l'unité dialectique du mouvement de l'apparaître dans sa double articulation.

La dialectique peut ainsi être considérée comme la structure interne du devenir aussi bien cosmologique qu'historique, à la condition qu'elle ne soit pas conçue comme une loi étrangère au temps. Au lieu de refléter une ratio extérieure au monde et à l'histoire, le mouvement dialectique est montré comme la structure même de la phénoménalité ${ }^{61}$. Si la dialectique ne vise plus la détermination des causes et ne s'identifie pas à un principe métaphysique - comme c'est le cas pour la dialectique idéelle et matérielle elle ne trouve sa légitimité qu'en tant que description des phénomènes : car, pour toute véritable phénoménologie, le phénomène précède la cause ${ }^{62}$.

Conçue comme dynamique interne à tout mouvement, à savoir à tout phénomène, la dialectique rend compte de l'articulation entre totalité physique et totalité historique. Hegel a tenté de concilier totalité et liberté au moyen d'un système rationnel et absolu, alors que Patočka, allant " par-delà Hegel, avec Hegel » ${ }^{63}$, articule une philosophie de la liberté et une phénoménologie de la totalité sans les ramener à un système logique achevé et $\operatorname{clos}^{64}$. La transposition phénoménologique du concept de dialectique hégélienne refuse ainsi la logique métaphysique de la réconciliation. C'est là, à nos yeux, le grand discrimen entre la dialectique spéculative hégélienne et la dialectique phénoménologique que Patočka esquisse : la dynamisation de l'être et de l'existence sous le signe d'un élément négatif-possibilisant détermine la sortie définitive de toute 
philosophie positive et systématique, pour revenir à une pensée critique et par là indéfiniment ouverte, prenant en charge la totalité des dimensions de l'être.

\section{NOTES}

1. M. Merleau-Ponty, Résumés de cours. Collège de France, 1952-1960, Paris, Gallimard, 1968, p. 109.

2. Cf. en particulier les essais de R. Barbaras, Le mouvement de l'existence. Études sur la phénoménologie de Jan Patočka, Chatou, Les Éditions de la Transparence, 2007 et L'ouverture du monde. Lecture de Jan Patočka, Chatou, Les Éditions de la Transparence, 2011. Voir aussi D. Duicu, Phénoménologie du mouvement: Patočka et l'héritage de la physique aristotélicienne, Paris, Vrin, 2015.

3. J. Patočka, Éternité et historicité, trad. par E. Abrams, Lagrasse, Verdier, 2011, p. 129.

4. J. Patočka, Le monde naturel et le mouvement de l'existence humaine, Dordrecht/Boston/Londres, Kluwer Academic Publishers, 1988, p. 62.

5. J. Patočka, Aristote, ses devanciers, ses successeurs, trad. par E. Abrams, Paris, Vrin, 2012, p. 403-404.

6. Les ouvrages de Patočka qui témoignent de manière explicite de ce dialogue sont nombreux. $\mathrm{Au}$ cours des années 1940 et 1950: le manuscrit inédit Hegelova "Fenomenologie ducha", Přednáškový cyklus na FF UK 1949/1950, 3306-I, 1/III, Archiv Jana Patočky, contenant les notes du cours donné par Patočka à l'Université Charles de Prague ; un autre manuscrit, qui opère une critique parallèle de l'idéalisme transcendantal husserlien et de l'idéalisme absolu hégélien: Kritika Husserlovy fenomenologické filosofie, MS A/5 = 3000/024, 1950, Archiv Jana Patočky ; trad. par H. Blaschek-Hahn et K. Novotny, "Kritik der Husserlschen phänomenologischen Philosophie", in Vom Erscheinen als solchem. Texte aus dem Nachlaß, Freiburg/München, Alber, 2000. Des années 1960 : outre l'ouvrage déjà évoqué, Aristote, ses devanciers, ses successeurs, les essais réunis dans L'art et le temps, trad. par E. Abrams, Paris, POL, 1990. Ici, Patočka consacre à Hegel une réflexion qui s'étend des questions métaphysiques aux questions esthétiques (notamment dans sa préface à la traduction tchèque de l'Esthétique de Hegel: L'évolution philosophique et esthétique de Hegel).

7. La question s'avère d'autant plus légitime et intéressante si l'on pense aux réflexions de $\mathrm{M}$. Merleau-Ponty (mais aussi d'E. Fink) à ce sujet. Il suffit de se reporter à Les aventures de la dialectique et aux notes des cours donnés au Collège de France entre 1952 et 1960. La tâche du philosophe, dit Merleau-Ponty dans le résumé du cours sur le concept de nature, serait « d'élaborer un tel concept de l'être que les contradictions, ni acceptées, ni “dépassées", trouvent en lui leur place. Ce que les philosophies dialectiques modernes n'ont pas réussi à faire parce que la dialectique en elles restait encadrée dans une ontologie prédialectique, deviendrait possible pour une ontologie qui découvrirait dans l'être même un porte-à-faux ou un mouvement » (M. Merleau-Ponty, Résumés de cours, op. cit., p. 128).

8. J. Patočka, Éternité et historicité, op. cit., p. 129.

9. Sur les concepts de négativité et de transcendance négative, voir notamment $\mathrm{E}$. Tardivel, $\mathrm{La}$ liberté au principe. Essai sur la philosophie de Patočka, Paris, Vrin, 2011, p. 33 sq.

10. J. Patočka, Éternité et historicité, op. cit., p. 129.

11. J. Patočka, Le monde naturel et le mouvement de l'existence humaine, ${ }^{\text {op. cit., } \mathrm{p} .99 .}$

12. J. Patočka, Papiers phénoménologiques, trad. par E. Abrams, Grenoble, Jérôme Millon, 1995, p. 122. 
13. J. Patočka, Introduction à la phénoménologie de Husserl, trad. par E. Abrams, Grenoble, Jérôme Millon, 2002, p. 16.

14. Sur le sens du mot archè ainsi que sur le concept de physis cf. voir aussi M. Heidegger, « De l'essence et du concept de Physis ", trad. par F. Fédier, in Questions II, Paris, Gallimard, 1968.

15. J. Patočka, Le monde naturel et le mouvement de l'existence humaine, op. cit., p. 103.

16. Ibid., p. 129.

17. Sur l'interprétation patočkienne d'Aristote, cf. C. V. Spaak, O. Stanciu (éd.), Patočka lecteur d'Aristote. Phénoménologie, ontologie, cosmologie, Paris, Le Cercle Herméneutique, 2015.

18. J. Patočka, Papiers phénoménologiques, op. cit., p. 107.

19. Cf. J. Patočka, Aristote, ses devanciers, ses successeurs, op. cit., p. 173.

20. Cf. J. Patočka, Papiers phénoménologiques, op. cit., p. 40.

21. Idem.

22. Ibid., p. 107.

23. J. Patočka, Le monde naturel et le mouvement de l'existence humaine, op. cit., p. 127.

24. Cf. J. Patočka, Aristote, ses devanciers, ses successeurs, op. cit., p. 156.

25. Ibid., p. 149.

26. J. Patočka, Papiers phénoménologiques, op. cit., p. 40.

27. Cf. J. Patočka, Aristote, ses devanciers, ses successeurs, op. cit., p. 268.

28. Ibid., p. 41.

29. J. Patočka, Le monde naturel et le mouvement de l'existence humaine, op. cit., p. 136.

30. J. Patočka, Aristote, ses devanciers, ses successeurs, op. cit., p. 41.

31. J. Patočka, Le monde naturel et le mouvement de l'existence humaine, op. cit., p. 136.

32. J. Patočka, Aristote, ses devanciers, ses successeurs, op. cit., p. 403-404.

33. J. Patočka, Hegelova "Fenomenologie ducha", op. cit., p. 66.

34. J. Patočka, Aristote, ses devanciers, ses successeurs, op. cit., p. 403.

35. Cf. J. Patočka, «Lettres à Robert Campbell (1946-1950) ", in Les Temps Modernes, $n^{\circ} 48$, Paris, 1992. Dans sa lettre du 28 octobre 1961, Patočka écrit que « le devenir et le mouvement qui est à l'origine de toutes nos expériences est lui-même impossible sans un devenir plus profond et plus élémentaire qui est, non mouvement dans l'expérience et dans le monde, mais devenir et mouvement $d u$ monde en tant que tel, devenir ontologique ».

36. A. Kojève, Introduction à la lecture de Hegel. Leçons sur la Phénoménologie de l'Esprit professées de 1933 à 1939 à l'Ecole des Hautes Études, réunies et publiées par Raymond Queneau, Paris, Gallimard, 1962, p. 453.

37. J. Patočka, Le monde naturel et le mouvement de l'existence humaine, op. cit., p. 129.

38. J. Patočka, Introduction à la phénoménologie de Husserl, op. cit., p. 16.

39. J. Patočka, Le monde naturel et le mouvement de l'existence humaine, ${ }^{\text {op. cit., }}$ p. 176.

40. Ibid., p. 175.

41. Ibid., p. 120.

42. Cf. sur cette question l'étude d'E. Tardivel, La liberté au principe, op. cit.

43. J. Patočka, Éternité et historicité, op. cit., p. 129.

44. J. Patočka, Le monde naturel et le mouvement de l'existence humaine, op. cit., p. 92.

45. Ibid., p. 44.

46. J. Srubar, "Phénoménologie asubjective, monde de la vie et humanisme », in Jan Patočka: philosophie, phénoménologie, politique, M. Richir, É. Tassin (éd.), Grenoble, Jérôme Million, 1992, p. 98.

47. J. Patočka, Le monde naturel et le mouvement de l'existence humaine, op. cit., p. 69.

48. Cf. E. Tardivel, « La subjectivité dissidente. Étude sur Patočka », in Studia Phaenomenologica, Jan Patocka and the European Heritage, 7, 2007, p. 435-463, p. 455. 
49. Cf. J. Patočka, Le monde naturel et le mouvement de l'existence humaine, op. cit., p. 105 : « Le cercle de l'existence (exister en vue de soi-même, en vue du mode de son être) contient toujours d'une certaine façon le cercle de la vie qui accomplit les fonctions vitales afin de faire retour en ellemême et de revenir à elle-même ».

50. J. Patočka, Papiers phénoménologiques, op. cit., p. 122.

51. Cf. J. Patočka, Kritika Husserlovy fenomenologické filosofie, op. cit., p. 5.

52. J. Patočka, "Considérations sur La nostalgie de la Grèce à l'aube de l'idéalisme allemand ", in Phénoménologie et Politique. Mélanges offerts à J. Taminiaux, Bruxelles, Ousia, 1990, p. 505.

53. Idem.

54. Ibid., p. 509.

55. J. Patočka, Le monde naturel et le mouvement de l'existence humaine, op. cit., p. 62.

56. Idem.

57. Cf. J. Patočka, Essais hérétiques sur la philosophie de l'histoire, trad. par E. Abrams, Lagrasse, Verdier, 1990, p. 232 : la dialectique est « un problème vivant dans la mesure où elle nous aide à voir, à lire, à expliciter les phénomènes ".

58. Cf. R. Barbaras, L'ouverture du monde, op. cit., p. 250.

59. E. Tardivel, « La subjectivité dissidente », in Studia Phaenomenologica, op. cit., p. 455.

60. J. Patočka, «Platonisme négatif », in Liberté et sacrifice, trad. par E. Abrams, Grenoble, Jérôme Millon, 1990, p. 72.

61. Nous voudrions remercier ici Clément Bertot pour ses remarques soulignant la continuité entre Patočka et Hegel, notamment au sujet de la négativité et de l'histoire : suggérant l'idée d'une "logique du négatif " soustraite à toute totalisation conceptuelle, il nous a aidé à faire émerger une certaine légitimité de cette dialectique non métaphysique dans le champ de la philosophie de l'histoire. C'est ce que Patočka semble vouloir dire en affirmant que la pensée métaphysique serait «anhistorique car elle ignore la dialectique » (J. Patočka, "Platonisme négatif ", in Liberté et sacrifice, op. cit., p. 55).

Nous sommes conscients qu'au cours des années 1970 Patočka critique sévèrement l'idée de dialectique sur le plan de la philosophie de l'histoire (Cf. en particulier les essais de cette période réunis dans $\mathrm{J}$. Patočka, Liberté et sacrifice, op. cit.). Il nous semble pourtant que - comme nous avons essayé de le montrer tout au long de ces pages - au moins jusqu'aux années soixante, il ne cesse au contraire de découvrir une dialectique propre à l'apparaître et à l'existence ; et cela peut-être «malgré soi » - pour reprendre la formule de Merleau-Ponty - c'est-à-dire malgré la lourde dette métaphysique de ce concept.

62. Cf. J. Patočka, Essais hérétiques sur la philosophie de l'histoire, op. cit., p. 230 sq.

63. J. Patočka, Hegelova "Fenomenologie ducha", op. cit., p. 8.

64. Nous avons utilisé ailleurs l'expression « hégélianisme négatif " pour qualifier l'approche patočkienne de la pensée de Hegel, mettant en œuvre une véritable Aufhebung de son système métaphysique ( $C f$. notre livre, Jan Patočka. Dalla libertà alla natura, Macerata, EUM, 2019 [à paraître]). 